\title{
Bounds on the Spectral Radius of a Nonnegative Matrix and Its Applications
}

\author{
Danping Huang and Lihua You \\ School of Mathematical Sciences, South China Normal University, Guangzhou 510631, China \\ Correspondence should be addressed to Lihua You; ylhua@scnu.edu.cn
}

Received 3 July 2016; Accepted 15 September 2016

Academic Editor: Ali R. Ashrafi

Copyright (c) 2016 D. Huang and L. You. This is an open access article distributed under the Creative Commons Attribution License, which permits unrestricted use, distribution, and reproduction in any medium, provided the original work is properly cited.

We obtain the sharp bounds for the spectral radius of a nonnegative matrix and then obtain some known results or new results by applying these bounds to a graph or a digraph and revise and improve two known results.

\section{Introduction}

First we recall some basic definitions and notations that will be used in this paper. Let $A$ be an $n \times n$ real matrix and $\lambda_{1}, \lambda_{2}, \ldots, \lambda_{n}$ be the eigenvalues of $A$. Since $A$ is not symmetric in general, the eigenvalues may be complex numbers. Without loss of generality, we assume that $\left|\lambda_{1}\right| \geq\left|\lambda_{2}\right| \geq \cdots \geq$ $\left|\lambda_{n}\right|$, and then the spectral radius of $A$ is defined as $\rho(A)=$ $\left|\lambda_{1}\right|$; that is, it is the largest modulus of the eigenvalues of $A$. By the Perron-Frobenius theorem, we have the following: (1) $\rho(A)$ is an eigenvalue of $A$ if $A$ is a nonnegative matrix; (2) $\rho(A)=\lambda_{1}$ is simple if $A$ is a nonnegative irreducible matrix.

Let $G=(V, E)(\vec{G}=(V, E))$ be a graph (digraph) with vertex set $V=V(G)(=V(\vec{G}))=\left\{v_{1}, v_{2}, \ldots, v_{n}\right\}$ and edge set $E=E(G)(\operatorname{arcset} E=E(\vec{G}))$. A graph $G($ digraph $\vec{G})$ is simple if it has no loops and multiple edges (arcs). For any pairs of vertices $v_{i}, v_{j} \in V$, if there is a (directed) path from $v_{i}$ to $v_{j}$, the graph $G$ (digraph $\vec{G}$ ) is called (strongly) connected. In this paper, we consider finite, simple graphs and digraphs.

Let $G$ be a graph and $\operatorname{diag}(G)=\operatorname{diag}\left(d_{1}, d_{2}, \ldots, d_{n}\right)$ be the diagonal matrix of vertex degrees of $G$, where $d_{i}$ is the degree of vertex $v_{i}$.

Let $\vec{G}$ be a digraph; $N_{\vec{G}}^{-}\left(v_{i}\right)=\left\{v_{j} \in V(\vec{G}) \mid\left(v_{j}, v_{i}\right) \in\right.$ $E(\vec{G})\}$ and $N_{\vec{G}}^{+}\left(v_{i}\right)=\left\{v_{j} \in V(\vec{G}) \mid\left(v_{i}, v_{j}\right) \in E(\vec{G})\right\}$ denote the in-neighbors and out-neighbors of $v_{i}$, respectively. Let $d_{i}^{-}=\left|N_{\vec{G}}^{-}\left(v_{i}\right)\right|$ and $d_{i}^{+}=\left|N_{\vec{G}}^{+}\left(v_{i}\right)\right|$ denote the indegree and outdegree of the vertex $v_{i}$ in $\vec{G}$, respectively, and $\operatorname{diag}(\vec{G})=$ $\operatorname{diag}\left(d_{1}^{+}, d_{2}^{+}, \ldots, d_{n}^{+}\right)$be the diagonal matrix of the vertex outdegrees of $\vec{G}$.

Let $A(G)=\left(a_{i j}\right)$ be the $(0,1)$ adjacency matrix of $G$, where

$$
a_{i j}= \begin{cases}1, & \text { if } v_{i} \text { and } v_{j} \text { are adjacent; } \\ 0, & \text { otherwise. }\end{cases}
$$

Let $A(\vec{G})=\left(a_{i j}\right)$ denote the adjacency matrix of $\vec{G}$, where $a_{i j}$ is equal to the number of $\operatorname{arcs}\left(v_{i}, v_{j}\right)$.

Then the signless Laplacian matrix of $G(\vec{G})$ is defined as

$$
\begin{gathered}
Q(G)=\operatorname{diag}(G)+A(G) \\
(Q(\vec{G})=\operatorname{diag}(\vec{G})+A(\vec{G})) .
\end{gathered}
$$

The spectral radii of $A(G)$ and $Q(G)(A(\vec{G})$ and $Q(\vec{G}))$, denoted by $\rho(G)$ and $q(G)(\rho(\vec{G})$ and $q(\vec{G}))$, are called the (adjacency) spectral radius of $G(\vec{G})$ and the signless Laplacian spectral radius of $G(\vec{G})$, respectively.

Let $G=(V, E)$ be a connected graph and $\vec{G}=(V, E)$ be a strong connected digraph. For $u, v \in V$, the distance from $u$ to $v$, denoted by $d_{G}(u, v)\left(d_{\vec{G}}(u, v)\right)$, is the length of the shortest (directed) path from $u$ to $v$ in $G(\vec{G})$. For $u \in V$, the transmission of vertex $u$ in $G(\vec{G})$ is the sum of distances from $u$ to all other vertices of $G(\vec{G})$, denoted by $\operatorname{Tr}_{G}(u)\left(\operatorname{Tr}_{\vec{G}}(u)\right)$. 
The distance matrix of $G(\vec{G})$ is the $n \times n$ matrix $\mathscr{D}(G)=$ $\left(d_{i j}\right)$, where $d_{i j}=d_{G}\left(v_{i}, v_{j}\right)\left(\mathscr{D}(\vec{G})=\left(d_{i j}\right)\right.$, where $d_{i j}=$ $\left.d_{\vec{G}}\left(v_{i}, v_{j}\right)\right)$. In fact, for $1 \leq i \leq n$, the transmission of vertex $v_{i}, \operatorname{Tr}_{G}\left(v_{i}\right)\left(\operatorname{Tr}_{\vec{G}}\left(v_{i}\right)\right)$, is just the $i$ th row sum of $\mathscr{D}(G)(\mathscr{D}(\vec{G}))$. For convenience, we also call $\operatorname{Tr}_{G}\left(v_{i}\right)\left(\operatorname{Tr}_{\vec{G}}\left(v_{i}\right)\right)$ the distance degree (outdegree) of vertex $v_{i}$ in $G(\vec{G})$, denoted by $D_{i}\left(D_{i}^{+}\right)$; that is, $D_{i}=\sum_{j=1}^{n} d_{i j}=\operatorname{Tr}_{G}\left(v_{i}\right)\left(D_{i}^{+}=\sum_{j=1}^{n} d_{i j}=\operatorname{Tr}_{\vec{G}}\left(v_{i}\right)\right)$. Similarly, we define $D_{i}^{-}=\sum_{j=1}^{n} d_{j i}$.

Let $\operatorname{Tr}(G)=\operatorname{diag}\left(D_{1}, D_{2}, \ldots, D_{n}\right)$ be the diagonal matrix of vertex transmissions of $G$, and let $\operatorname{Tr}(\vec{G})=\operatorname{diag}\left(D_{1}^{+}, D_{2}^{+}\right.$, $\ldots, D_{n}^{+}$) be the diagonal matrix of vertex transmissions of $\vec{G}$. The distance signless Laplacian matrix of $G(\vec{G})$ is the $n \times n$ matrix defined by Aouchiche and Hansen as [1]

$$
\begin{gathered}
Q(G)=\operatorname{Tr}(G)+\mathscr{D}(G) \\
(\mathscr{Q}(\vec{G})=\operatorname{Tr}(\vec{G})+\mathscr{D}(\vec{G})) .
\end{gathered}
$$

The spectral radii of $\mathscr{D}(G)$ and $\mathscr{Q}(G)(\mathscr{D}(\vec{G})$ and $\mathscr{Q}(\vec{G}))$, denoted by $\rho^{\mathscr{D}}(G)$ and $q^{\mathscr{D}}(G)\left(\rho^{\mathscr{D}}(\vec{G})\right.$ and $\left.q^{\mathscr{D}}(\vec{G})\right)$, are called the distance spectral radius of $G(\vec{G})$ and the distance signless Laplacian spectral radius of $G(\vec{G})$, respectively.

Let $G$ be a connected graph. The reciprocal distance matrix (also called the Harary matrix) $R(G)=\left(r_{i j}\right)$ of $G$ is the $n \times n$ matrix, where $\left(r_{i j}\right)=1 / d_{i j}$ if $i \neq j$ and $r_{i i}=0$ for $i=1, \ldots, n$. Clearly, the reciprocal distance matrix $R(G)$ is nonnegative and symmetric.

Let $G$ be a graph and $\vec{G}$ be a digraph; we call $G(\vec{G})$ regular if each vertex of $G(\vec{G})$ has the same degree (outdegree). Other definitions, terminology, and notations not in the article can be found in [2-4].

In recent decades, there are many results on the bounds of the spectral radius of a nonnegative matrix and the various spectral radii of a graph or a digraph, including the spectral radius, the signless Laplacian spectral radius, the distance spectral radius, the distance signless Laplacian spectral radius, and the spectral radius of the reciprocal distance matrix; see [5-16] and so on.

In this paper, we obtain the sharp bounds for the spectral radius of a nonnegative (irreducible) matrix in Section 2 and then obtain some known results or new results by applying these bounds to a graph in Section 3 or a digraph in Section 4; we revise and improve two known results.

\section{Main Results}

In this section, we will obtain the sharp bounds for the spectral radius of a nonnegative (irreducible) matrix and revise and improve the result of Theorem 2.9 in [9]. The techniques used in this section are motivated by $[7,9,14]$ and so on.

Lemma 1 (see [2]). If $A$ is an $n \times n$ nonnegative matrix with the spectral radius $\lambda(A)$ and row sums $r_{1}, r_{2}, \ldots, r_{n}$, then $\min _{1 \leq i \leq n} r_{i} \leq \lambda(A) \leq \max _{1 \leq i \leq n} r_{i}$. Moreover, if $A$ is irreducible, then one of the equalities holds if and only if the row sums of $A$ are all equal.
Theorem 2. Let $A=\left(a_{i j}\right)$ be an $n \times n$ nonnegative matrix with row sums $r_{1}, r_{2}, \ldots, r_{n}$, where $r_{1} \geq r_{2} \geq \cdots \geq r_{n}$, and let $S$ be the smallest diagonal element, $T$ be the smallest nondiagonal element, and $\lambda(A)$ be the spectral radius of $A$. Take $\phi_{1}=r_{n}$ and for $2 \leq l \leq n$,

$\phi_{l}$

$$
=\frac{r_{n}+S-T+\sqrt{\left(r_{n}+T-S\right)^{2}+4(l-1)\left(r_{l-1}-r_{n}\right) T}}{2} .
$$

Let $\phi_{t}=\max _{1 \leq l \leq n}\left\{\phi_{l}\right\}$ for some $1 \leq t \leq n$. Then $\lambda(A) \geq \phi_{t}$. Moreover, if $A$ is irreducible, then

(1) $\lambda(A)=\phi_{1}=r_{n}$ if and only if $r_{1}=r_{2}=\cdots=r_{n}$.

(2) $\lambda(A)=\phi_{t}>r_{n}$ with $2 \leq t \leq n$ if and only if $A$ satisfies the following conditions:

(i) $a_{i i}=S$ for $1 \leq i \leq t-1$;

(ii) $a_{i j}=T>0$ for $1 \leq i \leq n, 1 \leq j \neq i \leq t-1$;

(iii) $r_{1}=r_{2}=\cdots=r_{t-1}>r_{t}=r_{t+1}=\cdots=r_{n}$.

Proof. If $T=0$, then $\phi_{l}=\phi_{1}=r_{n}$ for any $2 \leq l \leq n$ by $r_{n} \geq S$. Thus by Lemma 1 and $r_{1} \geq r_{2} \geq \cdots \geq r_{n}$, we have $\lambda(A) \geq r_{n}=$ $\max _{1 \leq l \leq n}\left\{\phi_{l}\right\}=\phi_{1}$, and if $A$ is irreducible, $\lambda(A)=\phi_{1}=r_{n}$ if and only if $r_{1}=r_{2}=\cdots=r_{n}$.

Now we consider the case $T>0$.

Firstly, we show $\lambda(A) \geq \phi_{l}$ for all $2 \leq l \leq n$.

Since $A$ is a nonnegative matrix, then $a_{p, q} \geq T>0$ for $1 \leq p \neq q \leq n$. Thus

$$
\sum_{j=1}^{l-1} a_{i j} \geq \begin{cases}S+(l-2) T, & \text { if } 1 \leq i \leq l-1 \\ (l-1) T, & \text { if } l \leq i \leq n\end{cases}
$$

Let

$$
=\frac{S-r_{n}+(2 l-3) T+\sqrt{\left(r_{n}+T-S\right)^{2}+4(l-1)\left(r_{l-1}-r_{n}\right) T}}{2(l-1) T} .
$$

It is easy to show that $x>1$. Take

$$
x_{j}= \begin{cases}x, & \text { if } 1 \leq j \leq l-1, \\ 1, & \text { if } l \leq j \leq n,\end{cases}
$$

and let $\mathbf{U}=\operatorname{diag}\left(x_{1}, x_{2}, \ldots, x_{n}\right)$ be a diagonal matrix of order $n$. Let $B=\mathbf{U}^{-1} A \mathbf{U}$, and then $B$ and $A$ have the same eigenvalues, and $\lambda(B)=\lambda(A)$.

Now we consider the row sums of $B$, say, $s_{1}, s_{2}, \ldots, s_{n}$.

Case $1(1 \leq i \leq l-1)$. Consider

$$
\begin{aligned}
s_{i} & =\sum_{j=1}^{n} \frac{x_{j}}{x_{i}} a_{i j}=\sum_{j=1}^{l-1} a_{i j}+\frac{1}{x} \sum_{j=l}^{n} a_{i j} \\
& =\frac{1}{x} \sum_{j=1}^{n} a_{i j}+\left(1-\frac{1}{x}\right) \sum_{j=1}^{l-1} a_{i j}=\frac{1}{x} r_{i}+\left(1-\frac{1}{x}\right) \sum_{j=1}^{l-1} a_{i j} \\
& \geq \frac{1}{x} r_{i}+\left(1-\frac{1}{x}\right)[S+(l-2) T]
\end{aligned}
$$




$$
\begin{aligned}
& =\frac{1}{x}\left(r_{i}-S\right)+S+\left(1-\frac{1}{x}\right)(l-2) T \\
& \geq \frac{1}{x}\left(r_{l-1}-S\right)+S+\left(1-\frac{1}{x}\right)(l-2) T,
\end{aligned}
$$

with equality if and only if (a) and (b) hold: (a) $a_{i i}=S$ and $a_{i j}=T$ if $1 \leq j \leq l-1$ with $j \neq i$ and (b) $r_{i}=r_{l-1}$.

Case $2(l \leq i \leq n)$. Consider

$$
\begin{aligned}
s_{i} & =\sum_{j=1}^{n} \frac{x_{j}}{x_{i}} a_{i j}=x \sum_{j=1}^{l-1} a_{i j}+\sum_{j=l}^{n} a_{i j} \\
& =\sum_{j=1}^{n} a_{i j}+(x-1) \sum_{j=1}^{l-1} a_{i j}=r_{i}+(x-1) \sum_{j=1}^{l-1} a_{i j} \\
& \geq r_{i}+(x-1)(l-1) T \geq r_{n}+(x-1)(l-1) T,
\end{aligned}
$$

with equality if and only if (c) and (d) hold: (c) $a_{i j}=T$ if $1 \leq j \leq l-1$ and (d) $r_{i}=r_{n}$.

Noting that

$$
\begin{aligned}
& r_{n}+(x-1)(l-1) T \\
& =\frac{1}{x}\left(r_{l-1}-S\right)+S+\left(1-\frac{1}{x}\right)(l-2) T \\
& =\frac{S+r_{n}-T+\sqrt{\left(r_{n}+T-S\right)^{2}+4(l-1)\left(r_{l-1}-r_{n}\right) T}}{2} \\
& =\phi_{l},
\end{aligned}
$$

then, by Lemma 1 , we have $\lambda(A)=\lambda(B) \geq \min \left\{s_{1}, s_{2}, \ldots\right.$, $\left.s_{n}\right\} \geq \phi_{l}$.

Noting that $\phi_{l} \geq \phi_{1}=r_{n}$ by $r_{n}+T \geq S$, thus $\lambda(A) \geq \phi_{t}$, where $\phi_{t}=\max _{1 \leq l \leq n}\left\{\phi_{l}\right\}$ for some $1 \leq t \leq n$. $n$.

Let $A$ be irreducible; $\phi_{t}=\max _{1 \leq l \leq n}\left\{\phi_{l}\right\}$ for some $1 \leq t \leq$

Case $1\left(\lambda(A)=\phi_{1}\right)$. For $2 \leq l \leq n$, by $\phi_{l} \geq \phi_{1}$ and $T>0$, we have $\phi_{l}=\phi_{1} \Leftrightarrow r_{l-1}=r_{n}$. Then

$$
\phi_{t}=\phi_{1} \Longleftrightarrow \phi_{l}=\phi_{1} \quad \forall 2 \leq l \leq n \Longleftrightarrow r_{1}=r_{2}=\cdots=r_{n} .
$$

On the other hand, by Lemma 1 and $r_{1} \geq r_{2} \geq \cdots \geq r_{n}$, we have

$$
\lambda(A)=r_{n} \Longleftrightarrow r_{1}=r_{2}=\cdots=r_{n} .
$$

By (11), (12), and $\phi_{1}=r_{n}$, (1) holds.

Case $2\left(\lambda(A)=\phi_{t}>\phi_{1}\right.$ for some $\left.2 \leq t \leq n\right)$. Then $r_{t-1}>r_{n}$ and $T>0$ by $\phi_{t}>\phi_{1}=r_{n}$.

If $\lambda(A)=\phi_{t}$, then $s_{1}=s_{2}=\cdots=s_{n}=\phi_{t}$ by the above arguments and Lemma 1; thus (a) and (b) hold for $1 \leq i \leq t-1$ and (c) and (d) hold for $t \leq i \leq n$. Thus $a_{i i}=S$ for $1 \leq i \leq$ $t-1, r_{1}=r_{2}=\cdots=r_{t-1}>r_{t}=r_{t+1}=\cdots=r_{n}$ and $a_{i j}=T>0$ for $1 \leq i \leq n, 1 \leq j \neq i \leq t-1$. Now (i), (ii), and (iii) follow.

Conversely, if (i), (ii), and (iii) hold, it is easy to show that equality holds.
Corollary 3. Let $A=\left(a_{i j}\right)$ be an $n \times n$ nonnegative matrix with row sums $r_{1}, r_{2}, \ldots, r_{n}$, where $r_{1} \geq r_{2} \geq \cdots \geq r_{n}$, and let $S$ be the smallest diagonal element, $T$ be the smallest nondiagonal element, and $\lambda(A)$ be the spectral radius of $A$. Take $\phi_{1}=r_{n}$ and, for $2 \leq l \leq n$,

$\phi_{l}$

$$
=\frac{r_{n}+S-T+\sqrt{\left(r_{n}+T-S\right)^{2}+4(l-1)\left(r_{l-1}-r_{n}\right) T}}{2} .
$$

Let $\phi_{t}=\max _{1 \leq l \leq n}\left\{\phi_{l}\right\}$ for some $1 \leq t \leq n$. Then $\lambda(A) \geq \phi_{t}$. Moreover, if $A$ is irreducible with $T=0$ or $A$ is irreducible and symmetric, then

$$
\lambda(A)=\phi_{t} \quad \text { iff } t=1, r_{1}=r_{2}=\cdots=r_{n} .
$$

Proof. We complete the proof by the following two cases.

Case $1(T=0)$. It is obvious by the proof of Theorem 2 .

Case $2(A$ is symmetric and $T>0)$. By (i) and (ii), $A$ is symmetric and $T$ is the smallest nondiagonal element. We have $r_{1}=r_{2}=\cdots=r_{t-1}=S+(n-1) T<r_{t}=\cdots=r_{n}$. It is a contradiction by the fact $r_{t-1} \geq r_{t}$.

Similar to the proof of Theorem 2 (so we omit the proof of Theorem 4), we can show Theorem 4 which revises and improves the result of Theorem 2.9 in [9].

Theorem 4. Let $A=\left(a_{i j}\right)$ be an $n \times n$ nonnegative matrix with row sums $r_{1}, r_{2}, \ldots, r_{n}$, where $r_{1} \geq r_{2} \geq \cdots \geq r_{n}$, and let $M$ be the largest diagonal element, $N$ be the largest nondiagonal element, and $\lambda(A)$ be the spectral radius of $A$. Take $\phi_{1}=r_{1}$ and, for $2 \leq l \leq n$,

$\phi_{l}$

$$
=\frac{r_{l}+M-N+\sqrt{\left(r_{l}+N-M\right)^{2}+4(l-1)\left(r_{1}-r_{l}\right) N}}{2} .
$$

Let $\phi_{t}=\min _{1 \leq l \leq n}\left\{\phi_{l}\right\}$ for some $1 \leq t \leq n$. Then $\lambda(A) \leq \phi_{t}$. Moreover, if $A$ is irreducible, then

(1) $\lambda(A)=\phi_{1}=r_{1}$ if and only if $r_{1}=r_{2}=\cdots=r_{n}$.

(2) $\lambda(A)=\phi_{t}<r_{1}$ with $2 \leq t \leq n$ if and only if $A$ satisfies the following conditions:

(i) $a_{i i}=M$ for $1 \leq i \leq t-1$;

(ii) $a_{i j}=N>0$ for $1 \leq i \leq n, 1 \leq j \neq i \leq t-1$;

(iii) $r_{1}=r_{2}=\cdots=r_{t-1}>r_{t}=r_{t+1}=\cdots=r_{n}$.

\section{Various Spectral Radii of a Graph}

Let $G$ be a graph. In Section 1, the (adjacency) matrix $A(G)$, the signless Laplacian matrix $Q(G)$, the distance matrix $\mathscr{D}(G)$ (if $G$ is connected), the distance signless Laplacian matrix Q $(G)$ (if $G$ is connected), the reciprocal distance matrix $R(G)$ (if $G$ is connected), the (adjacency) spectral radius $\rho(G)$, the signless Laplacian spectral radius $q(G)$, the distance spectral 
radius $\rho^{\mathscr{D}}(G)$, the distance signless Laplacian spectral radius $q^{\mathscr{D}}(G)$, and the spectral radius of the reciprocal distance matrix $\lambda(R(G))$ are defined. Now, in this section, we will apply Theorem 2, Corollary 3, and Theorem 4 to $A(G), Q(G)$, $\mathscr{D}(G), Q(G)$, and $R(G)$ and obtain some new results or known results.

3.1. Adjacency Spectral Radius of a Graph. Let $G$ be a graph. By applying Corollary 3 and Theorem 4 to the (adjacency) matrix $A(G)$ with $S=0, T=0, M=0, N=1$, and $r_{i}=d_{i}$ for any $1 \leq i \leq n$, we have the following.

Corollary 5. Let $G$ be a graph on $n$ vertices with degree sequence $d_{1}, d_{2}, \ldots, d_{n}$, where $d_{1} \geq d_{2} \geq \cdots \geq d_{n}$. Then one has

$$
\begin{aligned}
& d_{n} \leq \rho(G) \\
& \leq \min _{1 \leq i \leq n}\left\{\frac{d_{i}-1+\sqrt{\left(d_{i}+1\right)^{2}+4(i-1)\left(d_{1}-d_{i}\right)}}{2}\right\} .
\end{aligned}
$$

Moreover, if $G$ is connected, then the left equality holds if and only if $G$ is a regular graph, the right equality holds if and only if $G$ is a regular graph, or there exists some $t$ with $2 \leq t \leq n$ such that $G$ is a bidegreed graph with $d_{1}=\cdots=d_{t-1}=n-1>$ $d_{t}=\cdots=d_{n}$.

Remark 6. The left inequality in Corollary 5 can be obtained by Lemma 1 immediately, and the right inequality in Corollary 5 is the result of Theorem 2.2 in [13].

3.2. Signless Laplacian Spectral Radius of a Graph. Let $G$ be a graph. By applying Corollary 3 and Theorem 4 to the signless Laplacian matrix $Q(G)$ with $S=d_{n}, T=0, M=d_{1}, N=1$, and $r_{i}=2 d_{i}$ for any $1 \leq i \leq n$, we have the following.

Corollary 7. Let $G$ be a graph on $n$ vertices with degree sequence $d_{1}, d_{2}, \ldots, d_{n}$, where $d_{1} \geq d_{2} \geq \cdots \geq d_{n}$. Then one has

$$
\begin{aligned}
& 2 d_{n} \leq q(G) \\
& \leq \min _{1 \leq i \leq n}\left\{\frac{d_{1}+2 d_{i}-1+\sqrt{\left(2 d_{i}-d_{1}+1\right)^{2}+8(i-1)\left(d_{1}-d_{i}\right)}}{2}\right\} .
\end{aligned}
$$

Moreover, if $G$ is connected, then the left equality holds if and only if $G$ is a regular graph, the right equality holds if and only if $G$ is a regular graph, or there exists some $t$ with $2 \leq t \leq n$ such that $G$ is a bidegreed graph in which $d_{1}=\cdots=d_{t-1}=$ $n-1>d_{t}=\cdots=d_{n}$.
Remark 8. The left inequality in Corollary 7 can be obtained by Lemma 1 immediately, and the right inequality in Corollary 7 is the result of Theorem 3.2 in [15].

3.3. Distance Spectral Radius of a Graph. Let $G$ be a connected graph and $d$ be the diameter of $G$. Then the distance matrix $\mathscr{D}(G)=\left(d_{i j}\right)$ is nonnegative and symmetric. By applying Corollary 3 and Theorem 4 to the distance matrix $\mathscr{D}(G)$ with $S=0, T=1, M=0, N=d$, and $r_{i}=D_{i}$ for any $1 \leq i \leq n$, we note that $d_{21}=\cdots=d_{n 1}=d$ implies a contradiction. Then we have the following.

Corollary 9. Let $G$ be a connected graph on $n$ vertices and $d$ be the diameter of $G$, with distance degree sequence $D_{1}, D_{2}, \ldots, D_{n}$ such that $D_{1} \geq D_{2} \geq \cdots \geq D_{n}$. Let

$$
f(i)=\frac{D_{n}-1+\sqrt{\left(D_{n}+1\right)^{2}+4(i-1)\left(D_{i-1}-D_{n}\right)}}{2} .
$$

Then one has

$$
\begin{aligned}
& \max _{2 \leq i \leq n}\left\{D_{n}, f(i)\right\} \leq \rho^{\mathscr{D}}(G) \\
& \leq \min _{1 \leq i \leq n}\left\{\frac{D_{i}-d+\sqrt{\left(D_{i}+d\right)^{2}+4 d(i-1)\left(D_{1}-D_{i}\right)}}{2}\right\} .
\end{aligned}
$$

Moreover, one of the equalities holds if and only if $D_{1}=D_{2}=$ $\cdots=D_{n}$.

Remark 10. The right inequality in Corollary 9 is the result of Corollary 1.8 in [6].

By applying Theorem 2 and Corollary 3 to the distance matrix $\mathscr{D}(G)$ with $S=0, T=1$, and $r_{i}=D_{i}$ for $i=1,2, \ldots, n$, we have the following.

Corollary 11 (see [16, Theorem 2]). Let $G$ be a connected graph on $n$ vertices with distance degree sequence $D_{1}, D_{2}, \ldots, D_{n}$ such that $D_{1} \geq D_{2} \geq D_{i-1}>D_{i} \geq \cdots \geq D_{n}$ for some $2 \leq i \leq n$. Then

$$
\begin{aligned}
& \rho^{\mathscr{D}}(G) \\
& \quad>\frac{D_{n}-1+\sqrt{\left(D_{n}+1\right)^{2}+4(i-1)\left(D_{i-1}-D_{n}\right)}}{2} .
\end{aligned}
$$

3.4. Distance Signless Laplacian Spectral Radius of a Graph. Let $G$ be a connected graph and $d$ be the diameter of $G$. Then the distance matrix $Q(G)$ is nonnegative and symmetric. By applying Corollary 3 and Theorem 4 to the distance matrix Q(G) with $S=D_{n}, T=1, M=D_{1}, N=d$, and $r_{i}=2 D_{i}$ for $i=1,2, \ldots, n$, we note that $d_{21}=\cdots=d_{n 1}=d$ implies a contradiction. Then we have the following. 
Corollary 12. Let $G$ be a connected graph on $n$ vertices with distance degree sequence $D_{1}, D_{2}, \ldots, D_{n}$ such that $D_{1} \geq D_{2} \geq$ $\cdots \geq D_{n}$ and $d$ be the diameter of $G$. Let

$$
\begin{aligned}
& f(i)=\frac{3 D_{n}-1+\sqrt{\left(D_{n}+1\right)^{2}+8(i-1)\left(D_{i-1}-D_{n}\right)},}{2}, \\
& g(i) \\
& =\frac{D_{1}+2 D_{i}-d+\sqrt{\left(2 D_{i}-D_{1}+d\right)^{2}+8 d(i-1)\left(D_{1}-D_{i}\right)}}{2} .
\end{aligned}
$$

Then one has

$$
\max _{2 \leq i \leq n}\left\{2 D_{n}, f(i)\right\} \leq q^{\mathscr{D}}(G) \leq \min _{1 \leq i \leq n}\{g(i)\} .
$$

Moreover, one of the equalities holds if and only if $D_{1}=D_{2}=$ $\cdots=D_{n}$.

Remark 13. The right inequality in Corollary 12 is the result of Theorem 3.8 in [9].

By applying Theorem 2 and Corollary 3 to the distance matrix $\mathbb{Q}(G)$ with $S=D_{n}, T=1$, and $r_{i}=2 D_{i}$ for $i=$ $1,2, \ldots, n$, we have the following.

Corollary 14. Let $G$ be a connected graph on $n$ vertices with distance degree sequence $D_{1}, D_{2}, \ldots, D_{n}$ such that $D_{1} \geq D_{2} \geq$ $D_{i-1}>D_{i} \geq \cdots \geq D_{n}$ for some $2 \leq i \leq n$. Then $q^{\mathscr{D}}(G)>f(i)$.

3.5. Spectral Radius of the Reciprocal Distance Matrix. By applying Corollary 3 and Theorem 4 to the reciprocal distance matrix $R(G)$ with $S=0, T=1 / d, M=0, N=1$, and $r_{i}=R_{i}$ for $i=1, \ldots, n$, we have the following.

Corollary 15. Let $G$ be a connected graph on $n$ vertices, $d$ be the diameter of $G, R_{i}=\sum_{j=1}^{n} r_{i j}$, and the row sum sequence be $R_{1}, R_{2}, \ldots, R_{n}$ of $R(G)$ satisfying $R_{1} \geq R_{2} \geq \cdots \geq R_{n}$. Let

$f(i)$

$$
\begin{aligned}
& =\frac{R_{n}-1 / d+\sqrt{\left(R_{n}+1 / d\right)^{2}+(4 / d)(i-1)\left(R_{i-1}-R_{n}\right)}}{2}, \\
& g(i)=\frac{R_{i}-1+\sqrt{\left(R_{i}+1\right)^{2}+4(i-1)\left(R_{1}-R_{i}\right)}}{2} .
\end{aligned}
$$

Then

$$
\max _{2 \leq i \leq n}\left\{R_{n}, f(i)\right\} \leq \lambda(R(G)) \leq \min _{1 \leq i \leq n}\{g(i)\} .
$$

Moreover, the left equality holds if and only if $R_{1}=R_{2}=$ $\cdots=R_{n}$, and the right equality holds if and only if either
$R_{1}=R_{2}=\cdots=R_{n}$ or there exists some $t$ with $2 \leq t \leq n$ such that $G$ is a graph with $t-1$ vertices of degree $n-1$ and the remaining $n-t+1$ vertices have equal degree less than $n-1$.

Remark 16. The right inequality in Corollary 15 is the result (i) of Theorem 4 in [16].

\section{Various Spectral Radii of a Digraph}

Let $\vec{G}$ be a strong connected digraph. In Section 1 , the adjacency matrix $A(\vec{G})$, the signless Laplacian matrix $Q(\vec{G})$, the distance matrix $\mathscr{D}(\vec{G})$ (if $\vec{G}$ is connected), the distance signless Laplacian matrix $\mathbb{Q}(\vec{G})$ (if $\vec{G}$ is connected), the adjacency spectral radius $\rho(\vec{G})$, the signless Laplacian spectral radius $q(\vec{G})$, the distance spectral radius $\rho^{\mathscr{D}}(\vec{G})$, and the distance signless Laplacian spectral radius $q^{\mathscr{D}}(\vec{G})$ are defined. Now, in this section, we will apply Theorem 2, Corollary 3 , and Theorem 4 to $A(\vec{G}), Q(\vec{G}), \mathscr{D}(\vec{G})$, and $Q(\vec{G})$, obtain some new results or known results, and revise and improve the result of Theorem 2.5 in [11].

4.1. Adjacency Spectral Radius of a Digraph. Let $\vec{G}$ be a digraph. By applying Corollary 3 and Theorem 4 to the (adjacency) matrix $A(\vec{G})$ with $S=0, T=0, M=0, N=1$, and $r_{i}=d_{i}^{+}$for $i=1, \ldots, n$, we have the following.

Corollary 17. Let $\vec{G}$ be a digraph on $n$ vertices with outdegree sequence $d_{1}^{+}, d_{2}^{+}, \ldots, d_{n}^{+}$such that $d_{1}^{+} \geq d_{2}^{+} \geq \cdots \geq d_{n}^{+}$. Then one has

$$
\begin{aligned}
& d_{n}^{+} \leq \rho(\vec{G}) \\
& \leq \min _{1 \leq i \leq n}\left\{\frac{d_{i}^{+}-1+\sqrt{\left(d_{i}^{+}+1\right)^{2}+4(i-1)\left(d_{1}^{+}-d_{i}^{+}\right)}}{2}\right\} .
\end{aligned}
$$

Moreover, if $\vec{G}$ is a strong connected digraph, then the left equality holds if and only if $\vec{G}$ is a regular digraph, the right equality holds if and only if $\vec{G}$ is a regular digraph, or there exists some $t$ with $2 \leq t \leq n$ such that $\vec{G}$ is a bidegreed digraph with $d_{1}^{+}=\cdots=d_{t-1}^{+}>d_{t}^{+}=\cdots=d_{n}^{+}$and the indegrees $d_{1}^{-}=\cdots=d_{t-1}^{-}=n-1$.

4.2. Signless Laplacian Spectral Radius of a Digraph. Let $\vec{G}$ be a digraph. By applying Corollary 3 and Theorem 4 to the signless Laplacian matrix $Q(\vec{G})$ with $S=d_{n}^{+}, T=0, M=$ $d_{1}^{+}, N=1$, and $r_{i}=2 d_{i}^{+}$for $i=1, \ldots, n$, we have the following.

Corollary 18. Let $\vec{G}$ be a digraph on $n$ vertices with outdegree sequence $d_{1}^{+}, d_{2}^{+}, \ldots, d_{n}^{+}$such that $d_{1}^{+} \geq d_{2}^{+} \geq \cdots \geq d_{n}^{+}$. Then one has 


$$
2 d_{n}^{+} \leq q(\vec{G}) \leq \min _{1 \leq i \leq n}\left\{\frac{d_{1}^{+}+2 d_{i}^{+}-1+\sqrt{\left(2 d_{i}^{+}-d_{1}^{+}+1\right)^{2}+8(i-1)\left(d_{1}^{+}-d_{i}^{+}\right)}}{2}\right\} .
$$

Moreover, if $\vec{G}$ is a strong connected digraph, then the left equality holds if and only if $\vec{G}$ is a regular digraph, the right equality holds if and only if $\vec{G}$ is a regular digraph, or there exists some $t$ with $2 \leq t \leq n$ such that $\vec{G}$ is a bidegreed digraph with $d_{1}^{+}=\cdots=d_{t-1}^{+}>d_{t}^{+}=\cdots=d_{n}^{+}$and the indegrees $d_{1}^{-}=\cdots=d_{t-1}^{-}=n-1$.
Remark 19. The left inequality in Corollary 18 can be obtained by Lemma 1 immediately, and the right inequality in Corollary 18 revises and improves Proposition 20.

Proposition 20 (see [11, Theorem 2.5]). Let $\vec{G}$ be a strong connected digraph on $n$ vertices with outdegree sequence $d_{1}^{+}, d_{2}^{+}, \ldots, d_{n}^{+}$such that $d_{1}^{+} \geq d_{2}^{+} \geq \cdots \geq d_{n}^{+}$. Then one has

$$
q(\vec{G}) \leq \min _{1 \leq i \leq n}\left\{\frac{d_{1}^{+}+2 d_{i}^{+}-1+\sqrt{\left(2 d_{i}^{+}-d_{1}^{+}+1\right)^{2}+8(i-1)\left(d_{1}^{+}-d_{i}^{+}\right)}}{2}\right\} .
$$

Moreover, if $i=1$, the equality holds if and only if $\vec{G}$ is a regular digraph. If $2 \leq i \leq n$, the equality holds if and only if $\vec{G}$ is a regular digraph or a bidegreed digraph in which $d_{1}^{+}=d_{2}^{+}=$ $\cdots=d_{i-1}^{+}=n-1$ and $d_{i}^{+}=\cdots=d_{n}^{+}=\delta^{+}$.

The following example shows that the result of Proposition 20 is incorrect.
Example 21. Let $n \geq 5$ and $D_{1}$ is shown in Figure 1. For $D_{1}$, the outdegree sequence is $3=d_{1}^{+}>d_{2}^{+}=d_{3}^{+}=\cdots=d_{n}^{+}=2$ and the indegree $d_{1}^{-}=n-1$. We have $q\left(D_{1}\right)=3+\sqrt{3}$ by direct computation. It is clear that

$$
q\left(D_{1}\right)=3+\sqrt{3}=\min _{1 \leq i \leq n}\left\{\frac{d_{1}^{+}+2 d_{i}^{+}-1+\sqrt{\left(2 d_{i}^{+}-d_{1}^{+}+1\right)^{2}+8(i-1)\left(d_{1}^{+}-d_{i}^{+}\right)}}{2}\right\}
$$

4.3. Distance Spectral Radius of a Digraph. Let $\vec{G}$ be a strong connected digraph and $d$ be the diameter of $\vec{G}$. By applying Theorems 2 and 4 to the distance matrix $\mathscr{D}(\vec{G})$ with $S=0, T=$ $1, M=0, N=d$, and $r_{i}=D_{i}^{+}$for $i=1, \ldots, n$, we note that $d_{21}=\cdots=d_{n 1}=d$ implies a contradiction. Then we have the following.

Corollary 22. Let $\vec{G}$ be a strong connected digraph on $n$ vertices with distance outdegree sequence $D_{1}^{+}, D_{2}^{+}, \ldots, D_{n}^{+}$such that $D_{1}^{+} \geq D_{2}^{+} \geq \cdots \geq D_{n}^{+}$, and let d be the diameter of $\vec{G}$. Let

$$
=\frac{D_{n}^{+}-1+\sqrt{\left(D_{n}^{+}+1\right)^{2}+4(i-1)\left(D_{i-1}^{+}-D_{n}^{+}\right)}}{2},
$$

$g(i)$

$$
=\frac{D_{i}^{+}-d+\sqrt{\left(D_{i}^{+}+d\right)^{2}+4 d(i-1)\left(D_{1}^{+}-D_{i}^{+}\right)}}{2} .
$$

Then one has

$$
\max _{2 \leq i \leq n}\left\{D_{n}^{+}, f(i)\right\} \leq \rho^{\mathscr{D}}(\vec{G}) \leq \min _{1 \leq i \leq n}\{g(i)\}
$$

Moreover, the left equality holds if and only if $D_{1}^{+}=\cdots=D_{n}^{+}$ or there exists some $t$ with $2 \leq t \leq n$ such that $D_{1}^{+}=\cdots=$ $D_{t-1}^{+}>D_{t}^{+}=\cdots=D_{n}^{+}$and $D_{1}^{-}=\cdots=D_{t-1}^{-}=n-1$ and the right equality holds if and only if $D_{1}^{+}=\cdots=D_{n}^{+}$.

4.4. Distance Signless Laplacian Spectral Radius of a Digraph. Let $\vec{G}$ be a strong connected digraph and $d$ be the diameter of $\vec{G}$. By applying Theorems 2 and 4 to the distance signless Laplacian matrix $Q(\vec{G})$ with $S=D_{n}^{+}, T=1, M=D_{1}^{+}, N=d$, and $r_{i}=2 D_{i}^{+}$for $i=1, \ldots, n$, we note two facts: the first fact is that (i) and (iii) of (2) in Theorem 2 cannot hold at the same time by $a_{i i}=D_{i}^{+}=\sum_{1 \leq j \leq n} d_{i j}$ and $r_{i}=2 D_{i}^{+}$, and the second fact is that $d_{21}=\cdots=d_{n 1}=d$ implies a contradiction. Then we have the following. 


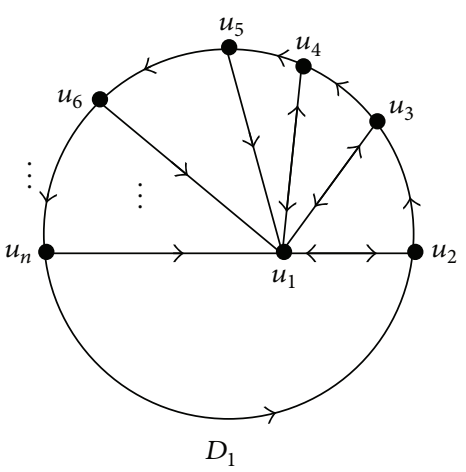

Figure 1: The digraphs $D_{1}$.

Corollary 23. Let $\vec{G}$ be a strong connected digraph on $n$ vertices with distance outdegree sequence $D_{1}^{+}, D_{2}^{+}, \ldots, D_{n}^{+}$such that $D_{1}^{+} \geq D_{2}^{+} \geq \cdots \geq D_{n}^{+}$, and let $d$ be the diameter of $\vec{G}$. Let

$$
\begin{aligned}
& f(i)=\frac{3 D_{n}^{+}-1+\sqrt{\left(D_{n}^{+}+1\right)^{2}+8(i-1)\left(D_{i-1}^{+}-D_{n}^{+}\right)},}{2}, \\
& g(i) \\
& =\frac{D_{1}^{+}+2 D_{i}^{+}-d+\sqrt{\left(2 D_{i}^{+}-D_{1}^{+}+d\right)^{2}+8 d(i-1)\left(D_{1}^{+}-D_{i}^{+}\right)}}{2} .
\end{aligned}
$$

Then one has

$$
\max _{2 \leq i \leq n}\left\{D_{n}^{+}, f(i)\right\} \leq q^{\mathscr{D}}(\vec{G}) \leq \min _{1 \leq i \leq n}\{g(i)\} .
$$

Moreover, one of the equalities holds if and only if $D_{1}^{+}=\cdots=$ $D_{n}^{+}$.

\section{Competing Interests}

The authors declare that there is no conflict of interests regarding the publication of this paper.

\section{Acknowledgments}

Lihua You's research is supported by the National Natural Science Foundation of China (Grant no. 11571123) and the Guangdong Provincial Natural Science Foundation (Grant no. 2015A030313377); Danping Huang's research is supported by the Scientific Research Foundation of Graduate School of South China Normal University (Grant no. 2015lkxm19). The authors would like to thank Yafei chen for the valuable comments, corrections, and suggestions, which lead to an improvement of the original paper.

\section{References}

[1] M. Aouchiche and P. Hansen, "Two Laplacians for the distance matrix of a graph," Linear Algebra and Its Applications, vol. 439, no. 1, pp. 21-33, 2013.

[2] A. Berman and R. J. Plemmons, Nonnegative Matrices in the Mathematical Sciences, Academic Press, New York, NY, USA, 1979.
[3] J. A. Bondy and U. S. R. Murty, Graph Theory with Applications, Macmillan, London, UK, 1976.

[4] D. Cvetković, P. Rowlinson, and S. Simić, An Introduction to the Theory of Graph Spectra, Cambridge University Press, Cambridge, UK, 2009.

[5] S. B. Bozkurt and D. Bozkurt, "On the signless Laplacian spectral radius of digraphs," Ars Combinatoria, vol. 108, pp. 193200, 2013.

[6] Y. Y. Chen, H. Q. Lin, and J. L. Shu, "Sharp upper bounds on the distance spectral radius of a graph," Linear Algebra and Its Applications, vol. 439, no. 9, pp. 2659-2666, 2013.

[7] X. Duan and B. Zhou, "Sharp bounds on the spectral radius of a nonnegative matrix," Linear Algebra and Its Applications, vol. 439, no. 10, pp. 2961-2970, 2013.

[8] C.-X. He, Y. Liu, and Z.-H. Zhao, "Some new sharp bounds on the distance spectral radius of graph," MATCH. Communications in Mathematical and in Computer Chemistry, vol. 63, no. 3, pp. 783-788, 2010.

[9] W. X. Hong and L. H. You, "Further results on the spectral radius of matrices and graphs," Applied Mathematics and Computation, vol. 239, pp. 326-332, 2014.

[10] W. Hong and L. You, "Spectral radius and signless Laplacian spectral radius of strongly connected digraphs," Linear Algebra and Its Applications, vol. 457, pp. 93-113, 2014.

[11] W. W. Lang and L. G. Wang, "Sharp bounds for the signless Laplacian spectral radius of digraphs," Applied Mathematics and Computation, vol. 238, pp. 43-49, 2014.

[12] A. D. Maden, K. C. Das, and A. S. Çevik, "Sharp upper bounds on the spectral radius of the signless Laplacian matrix of a graph," Applied Mathematics and Computation, vol. 219, no. 10, pp. 5025-5032, 2013.

[13] J. L. Shu and Y. R. Wu, "Sharp upper bounds on the spectral radius of graphs," Linear Algebra and Its Applications, vol. 377, pp. 241-248, 2004.

[14] L. H. You, Y. J. Shu, and P. Z. Yuan, "Sharp upper and lower bounds for the spectral radius of a nonnegative irreducible matrix and its applications," Linear and Multilinear Algebra, 2016.

[15] L. H. You, Y. J. Shu, and X. D. Zhang, "A sharp upper bound for the spectral radius of a nonnegative matrix and applications," Czechoslovak Mathematical Journal, vol. 66, no. 3, pp. 701-715, 2016.

[16] B. Zhou and N. Trinajstić, "Further results on the largest eigenvalues of the distance matrix and some distance-based matrices of connected (molecular) graphs," Internet Electronic Journal of Molecular Design, vol. 6, no. 12, pp. 375-384, 2007. 


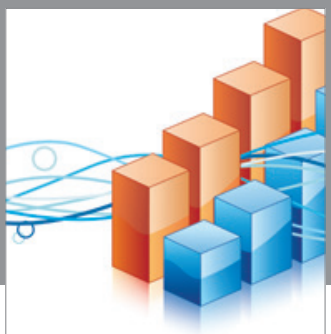

Advances in

Operations Research

vatem alat4

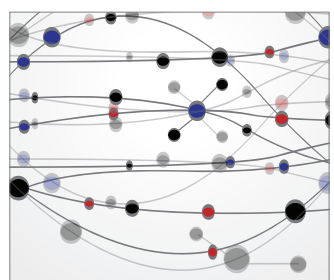

\section{The Scientific} World Journal
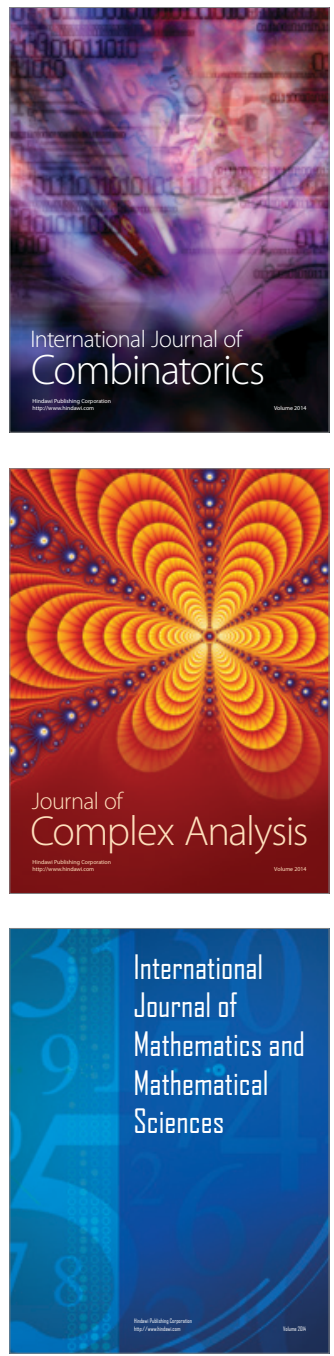
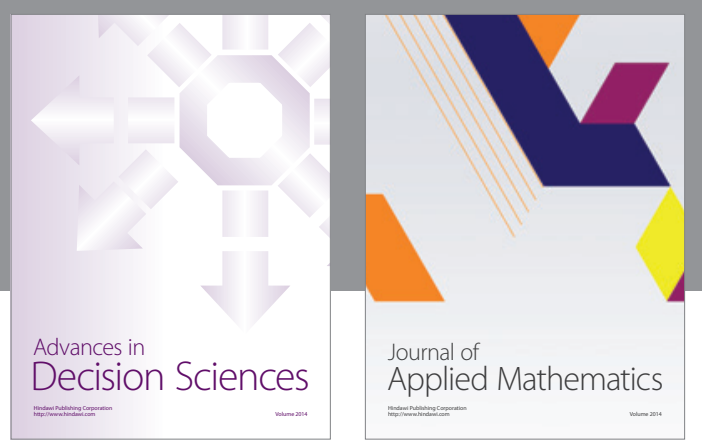

Algebra

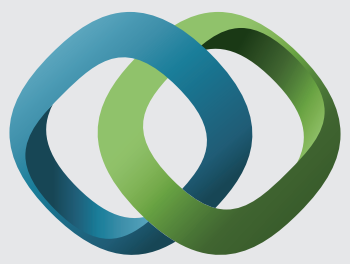

\section{Hindawi}

Submit your manuscripts at

http://www.hindawi.com
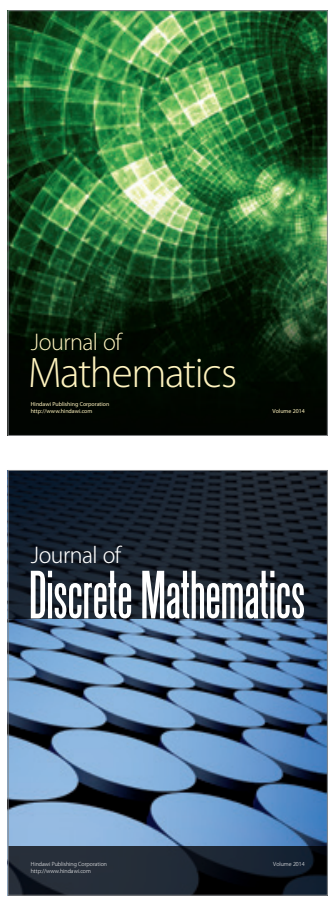

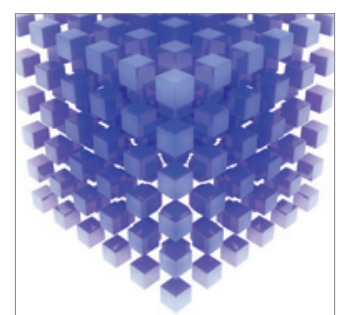

Mathematical Problems in Engineering
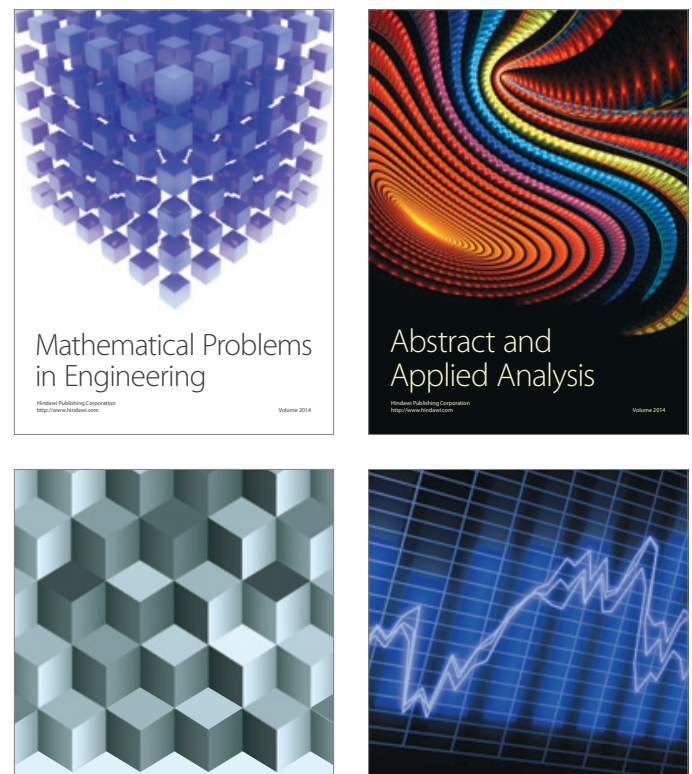

Journal of

Function Spaces

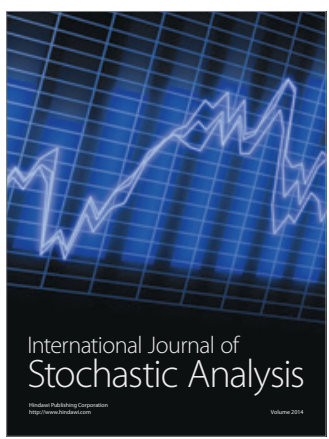

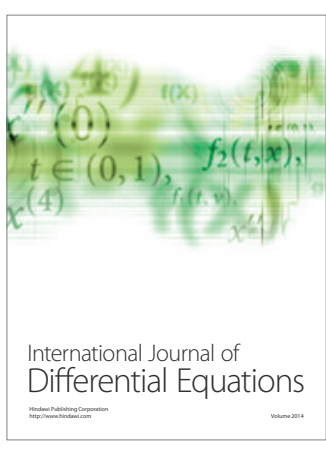
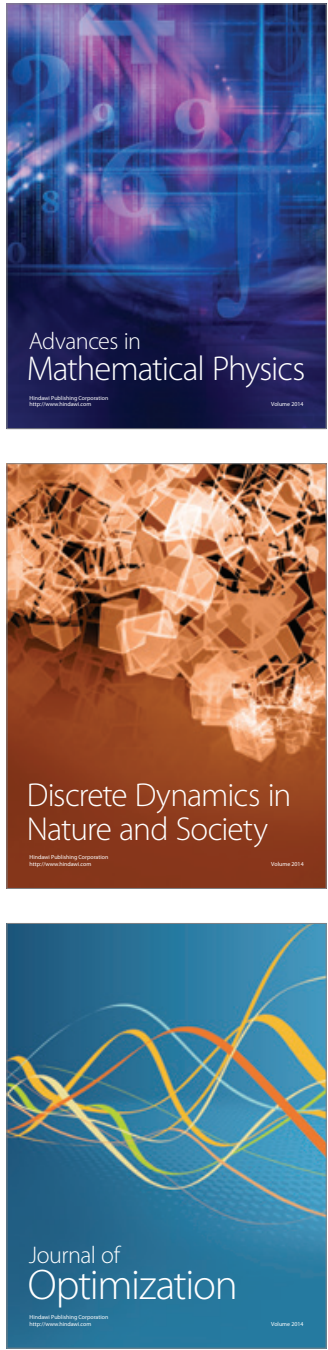